\title{
Cultural Adaptation of the Adolescent/Adult Sensory Profile for Spain
}

\author{
Berta Gándara-Gafo ${ }^{1}$, Sergio Santos-Del Riego ${ }^{2}$, Inés Viana-Moldes ${ }^{3}$, Javier \\ Muñiz ${ }^{4}$
}

${ }^{1}$ Berta Gándara-Gafo, PhD, OT, is Private Practitioner, Centro de Terapia Ocupacional Infantil TOIS, A Coruña, Spain; bertagandara@gmail.com.

${ }^{2}$ Sergio Santos-del Riego, PhD, is Professor, Radiology and Physical Medicine, Universidade da Coruña, A Coruña, Spain.

${ }^{3}$ Inés Viana-Moldes, BSc OT, BSc Psy, MSc, is PhD Student in Equity and Innovation in Education and Professor, Faculty of Health Sciences, Universidade da Coruña, A Coruña, Spain.

${ }^{4}$ Javier Muñiz, PhD, is Professor, University Institute of Health Sciences, University Biomedical Research Institute (INIBIC), Universidade da Coruña, A Coruña, Spain.

\begin{abstract}
Importance: In Spain, culturally adapted tools are needed to assess sensory processing in adolescents and adults.

Objective: To adapt the Adolescent/Adult Sensory Profile (AASP) so that it is culturally appropriate for use in Spain.

Design: Following standard procedures for cultural adaptation, we completed direct and back translation, conducted cognitive interviews, and examined test-retest reliability.

Participants: Cognitive interviews were conducted with 18 participants ages 11-13 yr. Test-retest reliability was calculated with different samples of 30 participants who were Spanish speaking only and 30 who were Spanish and English speaking.

Results: The cognitive interviews revealed no serious difficulties in comprehension. The linguistic expert made necessary adjustments, and test-retest reliability of items showed low intraclass correlation coefficients (ICCs; i.e., <.50) for two items in the monolingual group and five items in the bilingual group. These items were reviewed and revised by the linguistic expert, and subsequent test-retest reliability showed only one item with an ICC of $<.50$.

Conclusions and relevance: The cultural adaptation of the AASP for Spain is conceptually and semantically equivalent to the original version and provides a culturally sensitive measure of sensory processing for adolescent and adult Spanish populations.

What this article adds: The cultural adaptation of assessment tools is an essential part of occupational therapy clinical intervention. We describe the cultural adaptation to Spain of the Adolescent/Adult Sensory Profile.
\end{abstract}


Cultural diversity is a factor to be taken into account in scientific disciplines that study human interactions with the environment and in disciplines that focus on clinical practice (SuarezBalcazar et al., 2009). Sensory processing, a treatment approach used primarily in occupational therapy, has gained increased recognition in Spain; however, culturally sensitive sensory processing assessment tools have been lacking.

Studies in various countries have found differences in sensory processing in adult populations (i.e., Almomani et al., 2014; Chung, 2006), indicating the need for culturally adapted assessment tools that can identify these differences. The standard procedures to develop instruments for other languages and cultures follow two alternatives: (1) design new instruments or (2) adapt those that already exist.

Cultural adaptation has advantages over designing new tools: It reduces the cost of research, and it offers the possibility of carrying out comparative studies at the national and international levels using the same instrument (Hambleton \& Patsula, 1998), yielding benefits from the cumulative experience of many researchers. This approach can enrich the exchange of information within the scientific community, adding validity and coherence to results.

The International Test Commission (ITC; 2005), together with the American Educational Research Association (AERA), American Psychological Association, and National Council on Measurement in Education (1999), recommended a set of basic principles for carrying out cultural adaptations of assessment instruments with the aim of promoting a common methodology as a step toward universalizing protocols. These recommendations include direct and back translation; a cognitive comprehensibility interview, to ensure validity and cultural acceptability for the target population (Willis, 2005); and a reliability analysis, to determine the degree to which an instrument provides error-free measurements.

Reliability analysis of culturally adapted instruments includes test-retest reliability (i.e., the extent to which a tool consistently measures the same attribute at two different times in the same population), important in establishing the adapted instrument's equivalence to the original version. This analysis may be carried out in an exclusively monolingual target population or in a bilingual one, giving added value to the cultural adaptation (Carvajal et al., 2011).

The methods used in assessing sensory processing include standardized instruments such as proxy questionnaires that analyze sensory reactivity. Most of these instruments have been designed for children younger than age $12 \mathrm{yr}$, with only a few aimed at adolescent or adult populations, such as the Highly Sensitive Person Scale (Aron \& Aron, 1997), Adult Sensory Questionnaire (Kinnealey \& Oliver, 2002), Adolescent/Adult Sensory Profile (AASP; Brown \& Dunn, 2002), Sensory Responsiveness Questionnaire (Bar-Shalita et al., 2009), and Adult Sensory Processing Scale (Blanche et al., 2014).

The Sensory Profile (Brown \& Dunn, 2002; Dunn, 1999, 2002) questionnaires are used to analyze alterations in sensory reactivity. In Spain, occupational therapists have access to the cultural adaptation for Spain of the Short Sensory Profile (SSP; Beaudry \& Lane, 2015), designed for children between ages 3 and $10 \mathrm{yr}$. However, they do not have the necessary tools for older populations. Therefore, the aim of this study was to culturally adapt the AASP for Spain while maintaining the conceptual and semantic equivalence of the original version, thereby providing an instrument that identifies sensory reactivity issues in people age $11 \mathrm{yr}$ and older. 
Method

\section{Instrument}

The AASP, used for people age $11 \mathrm{yr}$ and older and originally created in the United States, has measurement characteristics and standard values designed for the U.S. population. It consists of 60 items divided into six sensory processing factors (taste/smell, movement, visual, tactile, activity level, and auditory processing) and four quadrants that reflect sensory patterns (low registration, sensation seeking, sensory sensitivity, and sensation avoiding). The internal consistency (Cronbach's $\alpha$ ) by quadrant is between .66 and .82 and was determined on the basis of a sample of 950 people without disabilities, divided into three age groups: $11-17 \mathrm{yr}, 18-64 \mathrm{yr}$, and $\geq 65 \mathrm{yr}$.

\section{Procedure}

The linguistic adaptation of the AASP followed the basic principles outlined by ITC (2005) and AERA et al. (1999), as well as the requirements stipulated in the research translation license agreement of NCS Pearson (Bloomington, MN), owner of the AASP. Our investigation was approved by the clinical research ethics committee of Galicia, Spain, and NCS Pearson. The anonymity of participants was preserved at all times during data collection.

\section{Direct Translation.}

For direct translation, we created a team of three bilingual translators, comprising a translator with dual citizenship (Spain and United States), an English philologist, and an occupational therapist. Spanish was the native language for all translators, and all of them had experience in technical text translation. The translator with dual citizenship considered cultural aspects of both countries to ensure reliability in cultural adaptation, and the occupational therapist certified conformity with the original material (Ramada-Rodilla et al., 2013).

Each expert worked independently and sent his or her translation of the instructions for use, items, and response options to the principal investigator (the first author), who compiled them and wrote the first draft of the questionnaire in coordination with the research team. The draft was then sent to the direct translation team, who evaluated the conceptual equivalence with the original (RománOyola \& Reynolds, 2010), indicating one of the following three options: (A) translation is equivalent, (B) translation is moderately equivalent with some linguistic but not semantic differences, and (C) translation is not equivalent. Justification and an alternative translation were requested for the items and instructions identified with $\mathrm{B}$ or $\mathrm{C}$, and they were revised until agreement was reached.

\section{Back Translation.}

Following the same methodology used in the direct translation process, the Spanish version was back translated by two new translators, per international recommendations (AERA et al., 1999; ITC, 2005): an American translator resident in Spain and a bilingual occupational therapist. When both translations were finished, the investigation team compared the original version and back-translations to analyze linguistic and semantic concordance and to detect any changes during the translation process. 


\section{Cognitive Interviews.}

Once the translation process was completed, individual semistructured cognitive interviews were carried out to qualitatively evaluate text comprehension and determine whether the vocabulary was adequate and culturally applicable (Ramada-Rodilla et al., 2013). According to Willis (2005), the samples used in cognitive interviewing should consist of between 5 and 15 people.

Participants, recruited through pediatricians in Asturias, Spain, met the following inclusion criteria: age 11-13 yr, Spanish nationality, and informed consent form signed by their legal guardian. Because Willis (2005) recommended the use of audio recording during interviews to later detect relevant information that could go unnoticed during an interview, we requested written consent from the parents for audio recordings.

Following the procedure used in similar investigations (Román-Oyola \& Reynolds, 2010), each participant received the AASP translation and, for each item, was asked to

- Give their opinion regarding comprehension;

- $\quad$ Paraphrase the item in their own words;

- Give a personal score;

- $\quad$ Provide verbal or body-language examples, or both; and

- $\quad$ Propose changes, if any, to improve text comprehension.

Items that presented comprehension difficulties for 2 or more participants were revised per recommended procedures (Willis, 2005).

\section{Linguistic Expert Review.}

The AASP translation was reviewed by a Spanish bilingual linguistic consultant to ensure cultural and grammatical accuracy. This expert analyzed the comprehension difficulties found during the cognitive interviews.

\section{Test-Retest Procedures.}

Test-retest procedures were used with two new samples to evaluate the quality of the translation, the cultural adaptation, and the viability of the adaptation. The following data were collected for each participant: gender, age, sociodemographic characteristics, educational level, and occupation. Two independent procedures were carried out: test-retest reliability of the Spanish version with Spanish-speaking participants $(n=30)$ and test-retest reliability using the Spanish version first and the English version second with bilingual participants ( $n=30$; Ramada-Rodilla et al., 2013). We considered participants to be bilingual if they had mastered both languages and could express themselves perfectly in each (Weinreich, 1979). To be included in the bilingual group, participants had to meet one of the following criteria: $2 \mathrm{yr}$ or more of residence in an English-speaking country, dual citizenship (Spanish-American, Spanish-British, Spanish-Australian, or SpanishCanadian), parents who were from an English-speaking country or who had dual citizenship (Spain and an English-speaking country), or academic qualification in the English language or in English translation or an official English proficiency certificate equivalent to an advanced level.

Test-retest reliability was evaluated by comparing scores on each item and quadrant by group (monolingual and bilingual) using the intraclass correlation coefficient (ICC; $p \leq .05$ was considered statistically significant). Items with ICC $<.50$ were revised, and a new test-retest procedure was carried out in the monolingual $(n=30)$ and bilingual $(n=30)$ samples. The data were analyzed using IBM SPSS Statistics (Version 19.0; IBM Corporation, Armonk, NY). 


\section{Results}

\section{Direct and Back Translation}

The direct translation analysis found that of a total of 60 items, 56 maintained similar vocabulary and composition, and 4 showed small differences. The investigation team brought together the direct translations and made small cultural adjustments in the use of vocabulary or in content that seemed odd in a Spanish context. An example of an adaptation is Item 7, "I don't like strongtasting mints or candies (for example, hot/cinnamon or sour candy)," translated as "No me gustan los caramelos de menta o caramelos con sabores fuertes (por ejemplo: caramelos picantes, de canela o ácidos)" and adapted for Spain as "No me gustan los caramelos o los dulces con sabores fuertes (por ejemplo: caramelos de menta, picantes o ácidos).,

The conceptual equivalence analysis of the 60 items concluded that $70 \%$ (42 items) were considered by the direct translation team to be equivalent (A); $25 \%$ (15 items) were considered moderately equivalent, with some linguistic but not semantic differences identified by at least one of the translators (B); and 5\% (3 items) were considered not equivalent according to one of the translators (C). Translator comments that were considered to aid in text comprehension and favor a better cultural adaptation to Spain were included in the cultural adaptation of the text.

In the back translations, the investigation team concluded that of the 60 items, $58(96.7 \%)$ maintained similar vocabulary and composition, and $2(2.3 \%)$ showed small semantic, but not linguistic, differences. In the conceptual analysis by the back translation team, 53 items $(88.3 \%)$ were determined to be conceptually equivalent (A) and $7(11.7 \%)$ to be moderately equivalent (B). Just as in direct translation, moderate conceptual equivalence resulted from adapting the text to Spanish culture. Translator comments that provided better text comprehension were included in the cultural adaptation. An example of an adaptation is Item 15, "I am unsure of footing when walking on stairs (for example, I trip, lose balance, and/or need to hold the rail)," translated as "No me siento seguro cuando camino por escaleras (por ejemplo: tropiezo, pierdo el equilibrio y/o tengo que apoyarme en el pasamanos)" and adapted for Spain as "No me siento seguro cuando subo o bajo por escaleras (por ejemplo: tropiezo, pierdo el equilibrio y/o tengo que apoyarme en el pasamanos)."

\section{Cognitive Interviews}

Eighteen participants between ages 11 and $13 \mathrm{yr}$ were interviewed. The group was distributed equally by gender and age. Of the 60 items, 38 were understood and accepted correctly; 14 were difficult for 1 of the participants; and 8 caused slight comprehension problems for 2 or more participants, with a need for subsequent revision. The results showed that the comprehension problems originated from the unclear use of words and phrasing. An example is Item 55, "I don't notice when my name is called," translated as "No me doy cuenta cuando me llaman," which caused difficulty for 4 participants, who did not know whether the sentence referred to when someone calls them by their name or calls them on the phone. Proposed changes included "No me doy cuenta cuando me llaman por la calle" and "No me doy cuenta cuando dicen mi nombre."

\section{Linguistic Expert Review}

The analysis of the linguistic expert led to modifications to the instructions of use, response options, and the items that caused problems in the cognitive interviews and to small modifications in other items to adapt them to Spanish linguistic norms. 


\section{Data Analysis}

Test-retest reliability was examined using samples of 30 monolingual participants with an average age of $38.1 \mathrm{yr}$ (standard deviation $[S D]=14.0$ ) and 30 bilingual participants with an average age of $33.5 \mathrm{yr}(S D=11.4)$. ICC values greater than .50 were found for 58 items in the monolingual sample and 55 items in the bilingual sample. A total of five items had ICCs of less than .50 in one or both samples. ICCs by quadrant were greater than .834 for both samples.

Although ICCs by quadrant were adequate, the five individual items with ICCs of less than .50 were revised by the principal investigator and linguistic expert and underwent an additional testretest procedure. Resulting correlations were between .585 and .955 for the monolingual sample and between .468 and .953 for the bilingual sample. These results show conceptual and semantic equivalence between the adapted questionnaire and the original one. Tables 1 and 2 show the final ICC results after the revision of the five items and the second test-retest procedure. Figures 1 and 2 show the Bland-Altman graphs (test-retest) for the monolingual and bilingual samples, respectively, showing scores close to 0 in the first and second measurement. 
Table 1. ICCs, by Item and by Quadrant, in the Monolingual Sample $(\mathrm{n}=30)$

\begin{tabular}{|c|c|c|c|c|c|c|c|c|c|c|c|}
\hline \multicolumn{3}{|c|}{ Low Registration } & \multicolumn{3}{|c|}{ Sensation Seeking } & \multicolumn{3}{|c|}{ Sensory Sensitivity } & \multicolumn{3}{|c|}{ Sensation Avoiding } \\
\hline No. & ICC & $95 \% \mathrm{CI}$ & No. & ICC & $95 \% \mathrm{CI}$ & No. & ICC & $95 \% \mathrm{CI}$ & No. & ICC & $95 \% \mathrm{CI}$ \\
\hline \multicolumn{12}{|c|}{ Item } \\
\hline 3 & $.929 * * *$ & {$[.852, .966]$} & 2 & $.876 * * *$ & {$[.739, .941]$} & 7 & $.792 * *$ & {$[.562, .901]$} & 1 & $.886^{* * *}$ & {$[.760, .946]$} \\
\hline 6 & $.792 * * *$ & {$[.563, .901]$} & 4 & $.585^{*}$ & {$[.129, .803]$} & 9 & $.900 * * *$ & {$[.791, .953]$} & 5 & $.719 * *$ & {$[.410, .866]$} \\
\hline 12 & $.925 * * *$ & {$[.846, .964]$} & 8 & $.821 * * *$ & {$[.624, .915]$} & 13 & $.825 * * *$ & {$[.633, .917]$} & 11 & $.891 * * *$ & {$[.772, .948]$} \\
\hline 15 & $.849 * * *$ & {$[.683, .928]$} & 10 & $.753 * * *$ & {$[.482, .883]$} & 16 & $.889 * * *$ & {$[.767, .947]$} & 18 & $.856^{* * *}$ & {$[.698, .932]$} \\
\hline 21 & $.771 * * *$ & {$[.520, .891]$} & 14 & $.703 * *$ & {$[.375, .858]$} & 20 & $.884 * * *$ & {$[.757, .945]$} & 24 & $.931 * * *$ & {$[.855, .967]$} \\
\hline 23 & $.821 * * *$ & {$[.625, .915]$} & 17 & $.810 * * *$ & {$[.601, .910]$} & 22 & $.940 * * *$ & {$[.873, .971]$} & 26 & $.817 * * *$ & {$[.616, .913]$} \\
\hline 36 & $.652 * *$ & {$[.269, .834]$} & 19 & $.778 * * *$ & {$[.534, .895]$} & 25 & $.895 * * *$ & {$[.779, .950]$} & 29 & $.843 * * *$ & {$[.670, .925]$} \\
\hline 37 & $.787 * * *$ & {$[.553, .899]$} & 28 & $.955 * * *$ & {$[.905, .978]$} & 27 & $.790 * * *$ & {$[.559, .900]$} & 35 & $.820 * * *$ & {$[.621, .914]$} \\
\hline 39 & $.632 * *$ & {$[.227, .825]$} & 30 & $.687 * *$ & {$[.343, .851]$} & 31 & $.858 * * *$ & {$[.702, .933]$} & 38 & $.816 * * *$ & {$[.614, .912]$} \\
\hline 41 & $.913 * * *$ & {$[.817, .959]$} & 32 & $.793 * * *$ & {$[.565, .901]$} & 33 & $.765 * * *$ & {$[.506, .888]$} & 43 & $.842 * * *$ & {$[.668, .925]$} \\
\hline 44 & $.890 * * *$ & {$[.770, .948]$} & 40 & $.708 * *$ & {$[.387, .861]$} & 34 & $.885 * * *$ & {$[.757, .945]$} & 46 & $.701^{* *}$ & {$[.371, .858]$} \\
\hline 45 & $.831 * * *$ & {$[.646, .920]$} & 42 & $.723 * * *$ & {$[.418, .868]$} & 48 & $.577 *$ & {$[.112, .799]$} & 49 & $.735^{* * *}$ & {$[.444, .874]$} \\
\hline 52 & $.879 * * *$ & {$[.746, .942]$} & 47 & $.761 * * *$ & {$[.498, .886]$} & 51 & $.882 * * *$ & {$[.751, .944]$} & 53 & $.856^{* * *}$ & {$[.697, .937]$} \\
\hline 55 & $.803 * * *$ & {$[.585, .906]$} & 50 & $.894 * * *$ & {$[.778, .950]$} & 54 & $.766 * * *$ & {$[.509, .889]$} & 56 & $.776 * * *$ & {$[.529, .893]$} \\
\hline 59 & $.880 * * *$ & {$[.747, .943]$} & 58 & $.743 * * *$ & {$[.461, .878]$} & 60 & $.789 * * *$ & {$[.557, .900]$} & 57 & $.852 * * *$ & {$[.688, .929]$} \\
\hline \multicolumn{12}{|c|}{ Quadrant } \\
\hline & $.925^{* * *}$ & {$[.842, .964]$} & & $.905^{* * *}$ & {$[.801, .951]$} & & $.933 * * *$ & {$[.859, .968]$} & & $.948^{* * * *}$ & {$[.891, .976]$} \\
\hline
\end{tabular}

Note. $\mathrm{CI}=$ confidence interval; $\mathrm{ICC}=$ intraclass correlation coefficient.

$* p<.05 . * * p<.01 . * * * p<.001$. 
Table 2. ICCs, by Item and by Quadrant, in the Bilingual Sample $(\mathrm{n}=30)$

\begin{tabular}{|c|c|c|c|c|c|c|c|c|c|c|c|}
\hline \multicolumn{3}{|c|}{ Low Registration } & \multicolumn{3}{|c|}{ Sensation Seeking } & \multicolumn{3}{|c|}{ Sensory Sensitivity } & \multicolumn{3}{|c|}{ Sensation Avoiding } \\
\hline No. & ICC & $95 \% \mathrm{CI}$ & No. & ICC & $95 \% \mathrm{CI}$ & No. & ICC & $95 \% \mathrm{CI}$ & No. & ICC & $95 \% \mathrm{CI}$ \\
\hline \multicolumn{12}{|c|}{ Item } \\
\hline 3 & $.737 * * *$ & {$[.448, .875]$} & 2 & $.746 * * *$ & {$[.466, .879]$} & 7 & $.916 * * *$ & {$[.823, .960]$} & 1 & $.695 * * *$ & {$[.359, .855]$} \\
\hline 6 & $.775 * * *$ & {$[.528, .893]$} & 4 & $.740 * * *$ & {$[.453, .876]$} & 9 & $.953 * * *$ & {$[.901, .978]$} & 5 & $.688 * * *$ & {$[.344, .851]$} \\
\hline 12 & $.858 * * *$ & {$[.701, .932]$} & 8 & $.827 * * *$ & {$[.636, .918]$} & 13 & $.699 * * *$ & {$[.367, .857]$} & 11 & $.930 * * *$ & {$[.852, .967]$} \\
\hline 15 & $.773 * * *$ & {$[.522, .892]$} & 10 & $.893 * * *$ & {$[.776, .949]$} & 16 & $.878 * * *$ & {$[.744, .942]$} & 18 & $.852 * * *$ & {$[.689, .930]$} \\
\hline 21 & $.725 * * *$ & {$[.422, .869]$} & 14 & $.799 * * *$ & {$[.578, .904]$} & 20 & $.881 * * *$ & {$[.750, .943]$} & 24 & $.827 * * *$ & {$[.636, .917]$} \\
\hline 23 & $.631 * *$ & {$[-.482, .664]$} & 17 & $.767 * * *$ & {$[.511, .889]$} & 22 & $.858 * * *$ & {$[.702, .932]$} & 26 & $.721 * * *$ & {$[.414, .867]$} \\
\hline 36 & $.779 * * *$ & {$[.536, .895]$} & 19 & $.830 * * *$ & {$[.644, .919]$} & 25 & $.900 * * *$ & {$[.789, .952]$} & 29 & $.567 * *$ & {$[.091, .794]$} \\
\hline 37 & $.929 * * *$ & {$[.851, .966]$} & 28 & $.668 * *$ & {$[-.498, .661]$} & 27 & $.799 * * *$ & {$[.578, .904]$} & 35 & $.862 * * *$ & {$[.710, .934]$} \\
\hline 39 & $.468 * *$ & {$[-.118, .747]$} & 30 & $.908 * * *$ & {$[.806, .956]$} & 31 & $.898 * * *$ & {$[.786, .952]$} & 38 & $.772 * * *$ & {$[.522, .892]$} \\
\hline 41 & $.849 * * *$ & {$[.682, .928]$} & 32 & $.935 * * *$ & {$[.863, .969]$} & 33 & $.857 * * *$ & {$[.699, .932]$} & 43 & $.836 * * *$ & {$[.654, .922]$} \\
\hline 44 & $.852 * * *$ & {$[.689, .930]$} & 40 & $.836 * * *$ & {$[.656, .922]$} & 34 & $.785^{* * *}$ & {$[.758, .898]$} & 46 & $.812 * * *$ & {$[.605, .910]$} \\
\hline 45 & $.828 * * *$ & {$[.639, .918]$} & 42 & $.862 * * *$ & {$[.710, .934]$} & 48 & $.874 * * *$ & {$[.736, .940]$} & 49 & $.844 * * *$ & {$[.643, .926]$} \\
\hline 52 & $.788 * * *$ & {$[.554, .899]$} & 47 & $.811 * * *$ & {$[.603, .910]$} & 51 & $.787 * * *$ & {$[.552, .898]$} & 53 & $.722 * * *$ & {$[.415, .867]$} \\
\hline 55 & $.598 * * *$ & {$[-.444, .673]$} & 50 & $.901 * * *$ & {$[.792, .953]$} & 54 & $.843 * * *$ & {$[.671, .925]$} & 56 & $.871 * * *$ & {$[.729, .939]$} \\
\hline 59 & $.713 * * *$ & {$[.397, .863]$} & 58 & $.883 * * *$ & {$[.754, .944]$} & 60 & $.737 * * *$ & {$[.448, .875]$} & 57 & $.773 * * *$ & {$[.524, .892]$} \\
\hline \multicolumn{12}{|c|}{ Quadrant } \\
\hline & $.907 * * *$ & {$[.806, .956]$} & & $.914 * * *$ & {$[.819, .959]$} & & $.942 * * *$ & {$[.879, .973]$} & & $.924 * * *$ & {$[.840, .96]$} \\
\hline
\end{tabular}

Note. CI $=$ confidence interval; ICC $=$ intraclass correlation coefficient.

$* p<.05 . * * p<.01 . * * * p<.001$. 

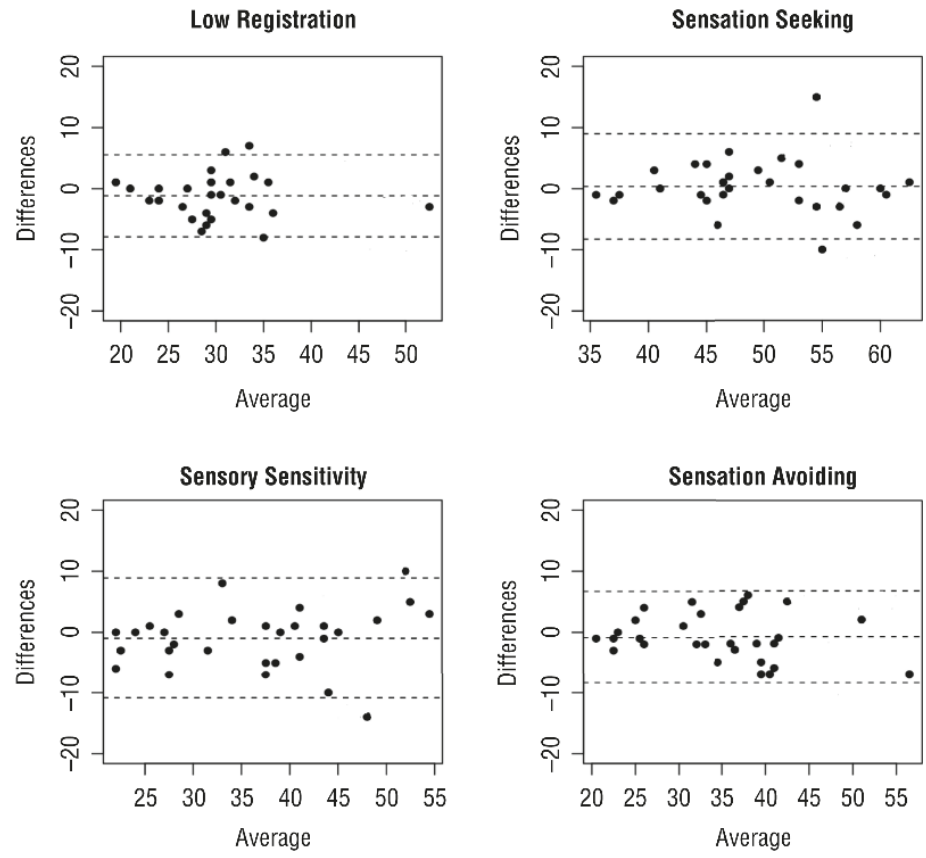

Figure 1. Bland-Altman graph (test-retest) for the monolingual sample $(n=30)$.
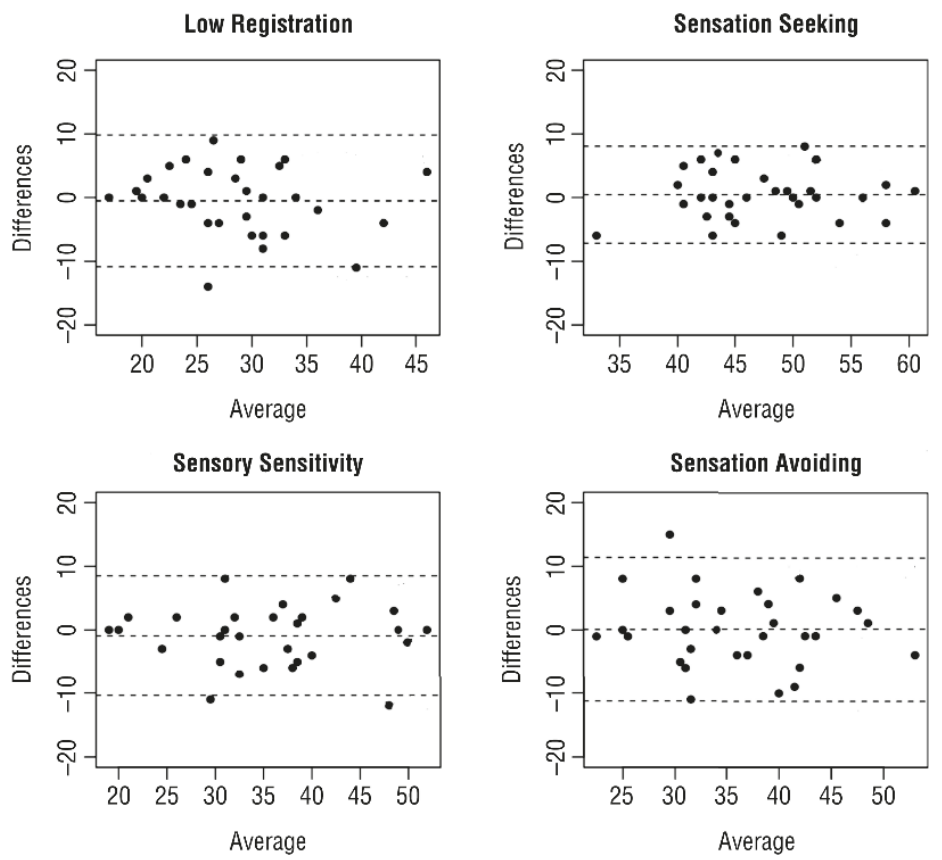

Figure 2. Bland-Altman graph (test-retest) for the bilingual sample $(n=30)$. 


\section{Discussion}

This work represents the first cultural adaptation for Spain of a tool for assessing sensory reactivity in adolescents and adults (Gándara-Gafo et al., 2016), specifically the AASP, an instrument used internationally in both clinical and investigative practice. When this project began, occupational therapists in Spain lacked sensory processing assessment tools that were culturally appropriate to the Spanish population. The growing desire of these therapists to identify and understand differences in sensory processing and to address these differences in their work first led to the cultural adaptation for Spain of the SSP (Beaudry \& Lane, 2015). This adaptation did not cover populations age $11 \mathrm{yr}$ and older, which made it necessary to design further adaptations that would allow longitudinal assessment of sensory processing across the life span in the Spanish population.

This work follows the guidelines of ITC (2005) and AERA et al. (1999). This methodology is accepted internationally in the cultural adaptation of assessment tools because it ensures maximum conceptual and semantic equivalence with the original version. The detailed direct and back translation work by bilingual professionals in direct contact with American and Spanish culture resulted in a translation reflecting common language use in Spain.

To ensure that the translation is suitable for the Spanish population as a whole, we conducted cognitive interviews with participants ages 11-13 yr, a group among whom greater comprehension difficulties might be expected. Cognitive interviews are increasingly used in cultural adaptation of questionnaires to identify and correct comprehension problems because they offer both feedback about the clarity of the text and evidence of validity regarding comprehension (Willis, 2005). This kind of procedure has been used in the cultural adaptation of the Sensory Profile for various ages; countries, such as Spain (Beaudry \& Lane, 2015) and Puerto Rico (Román-Oyola \& Reynolds, 2010).

Only eight items were identified as difficult by 2 or more participants and were subsequently revised by the linguistic expert and the principal investigator on the basis of participant feedback to ensure correct comprehension of the text. The comprehension difficulties were caused mainly by the use of words unfamiliar to the participants (e.g., for Item 1, use of sección or espacio for section; for Item 5, alimentos familiares for familiar foods; and for Item 46, multitudes for crowds). These problems may arise from the richness of synonyms in Spanish and the use of different words in certain population groups, depending on their age or cultural background. In addition, we observed that some comprehension problems stemmed from a lack of experience or an inadequate understanding of the literal meaning of the sentence.

As with the cultural adaptation for Spain of the SSP, this study included a review of the adapted AASP by a linguistic expert to ensure conformity to the grammatical and cultural features of the Spanish language used in Spain. Following previous investigations (Almomani et al., 2014; Chung, 2006; Ee et al., 2016; Neuman et al., 2004), once the translation of the text was finished, a repeatability (test-retest) analysis was carried out with the objective of evaluating both the quality of the Spanish version and the conceptual and semantic equivalence between the original and adapted versions. This study included test-retest procedures using both a monolingual and a bilingual sample, obtaining initially excellent ICC values, higher than .80 for the quadrants of this questionnaire and similar to the ones obtained in previous cultural adaptations of Sensory Profile tools (Chung, 2006). 
To improve linguistic, semantic, and cultural consistency and equivalence with the original version, two additional procedures were carried out with the five items with an ICC $<.50$. First, the items were reviewed and modified by both the principal investigator and the linguistic expert. Because three of these items $(23,39$, and 55) had negative grammatical structures in the original text, the recommendations of Beaton et al. (2000) were followed, and grammatical structure was altered to obtain an affirmative sentence to avoid false negatives and ambiguities in the answers. In addition, to clarify meaning, an example was added to Item 28 and the grammatical structure of Item 39 was modified.

Second, a new test-retest procedure was done for the five items with the monolingual and bilingual samples, and ICCs clearly improved. Only Item 39 for the bilingual sample still had a value $<.50$, probably as a result of problems of double negation between the original questionnaire and the adapted version. We decided that it was not appropriate to make any additional modification to Item 39 because it was difficult to find new wording that improved on the existing one. In addition, we chose not to omit the item because it would alter the applicability of the metrics of the original questionnaire. Given that the second test-retest procedure performed in this study to improve the cultural adaptation of the AASP was an additional procedure that it is not usually performed in other cultural adaptations, and given that the second test-retest procedure showed excellent scores (>.90) in all the quadrants for both samples, we did not deem it necessary to perform any additional procedures.

The results obtained in this study show that the cultural adaptation of the AASP for Spain maintains very good repeatability and stability of answers over time for both bilingual and monolingual participants. Therefore, professionals who use this assessment tool can rely on the stability of the results obtained in recording alterations in sensory reactivity in the Spanish population.

We suggest that the title of the Spanish version of the AASP should be modified to Perfil sensorial de los adolescentes, adultos y mayores because Spanish society does not usually characterize people age 65 and older with the term adult. With this change, professionals working in the area of sensory integration in Spain will be aware that this assessment tool is appropriate for the analysis of sensory reactivity in people from adolescence to the end of the life span.

\section{Implications for Occupational Therapy Practice}

The results of this research have the following implications for occupational therapy practice:

- $\quad$ The Spanish version of the AASP provides occupational therapy practitioners with the first comprehensive sensory processing assessment tool for adolescents and adults that is culturally adapted to and reliable for the Spanish population.

- The Spanish version of the AASP can contribute to the process of occupational therapy evaluation of Spanish adolescents and adults with sensory processing problems.

\section{Conclusion}

The results obtained in this study lead us to conclude that the Spanish version of the AASP is conceptually and semantically equivalent to the original American version. It is an adequate scale to measure sensory processing in the Spanish population age $11 \mathrm{yr}$ and older and is useful for occupational therapy practitioners as both a clinical and research tool. 


\section{Acknowledgments}

We thank Elena Castañón, Jonathan Mansilla, Natalia Rivas, Sara Arbolella, Tania Moriyón, and Isabelle Beaudry for their participation and to Jacinto Martínez and Cristina Suárez for their willingness and cooperation. We also thank the Scientific and Technical Services statistical consulting unit at the University of Oviedo and especially Tania Iglesias for her methodological advice. In addition, we thank the young people we interviewed, their families, and all the people who cooperated in the analysis of reproducibility and reliability. This research was carried out by the Integrated Health Research Unit (INTEGRA SAÚDE; University of A Coruña).

\section{References}

Almomani, F. M., Brown, C., Dahab, S. A., Almomani, M., \& Nadar, M. (2014). Cross cultural adaptation of the Adolescent/Adult Sensory Profile: Establishing linguistic equivalency and psychometric properties of the Arabic version. Disability and Rehabilitation, 36, 765-770. https://doi.org/10.3109/09638288.2013.819386

American Educational Research Association, American Psychological Association, \& National Council on Measurement in Education; Joint Committee on Standards for Educational and Psychological Testing. (1999). Standards for educational and psychological testing. Washington, DC: American Educational Research Association.

Aron, E. N., \& Aron, A. (1997). Sensory-processing sensitivity and its relation to introversion and emotionality. Journal of Personality and Social Psychology, 73, 345-368. https://doi.org/10.1037/00223514.73.2.345

Bar-Shalita, T., Seltzer, Z., Vatine, J. J., Yochman, A., \& Parush, S. (2009). Development and psychometric properties of the Sensory Responsiveness Questionnaire (SRQ). Disability and Rehabilitation, 31, 189201. https://doi.org/10.1080/09638280801903096

Beaton, D. E., Bombardier, C., Guillemin, F., \& Ferraz, M. B. (2000). Guidelines for the process of crosscultural adaptation of self-report measures. Spine, 25, 3186-3191. https://doi.org/10.1097/00007632200012150-00014

Beaudry, I. B., \& Lane, S. J. (2015). Cultural adaptation for Spain of the Spanish version of the Short Sensory Profile using cognitive interviews. Austin Journal of Autism and Related Disabilities, 1, 1004-1010.

Blanche, E. I., Parham, D., Chang, M., \& Mallinson, T. (2014). Development of an Adult Sensory Processing Scale (ASPS). American Journal of Occupational Therapy, 68, 531-538. https://doi.org/10.5014/ajot.2014.012484

Google Scholar

Brown, C., \& Dunn, W. (2002). Adolescent/Adult Sensory Profile: User's manual. San Antonio: Psychological Corporation.

Carvajal, A., Centeno, C., Watson, R., Martínez, M., \& Rubiales, A. S. (2011). Cómo validar un instrumento de medida de la salud? [How is an instrument for measuring health to be validated?] Anales del Sistema Sanitario de Navarra, 34, 63-72.

Chung, J. C. C. (2006). Measuring sensory processing patterns of older Chinese people: Psychometric validation of the adult Sensory Profile. Aging and Mental Health, 10, 648-655. https://doi.org/10.1080/13607860600648080

Dunn, W. (1999). Sensory Profile. San Antonio: Psychological Corporation.

Dunn, W. (2002). Infant/Toddler Sensory Profile. San Antonio: Psychological Corporation.

Ee, S. I., Loh, S. Y., Chinna, K., \& Marret, M. J. (2016). Cross-cultural adaptation and psychometric properties of the Malay version of the Short Sensory Profile. Physical and Occupational Therapy in Pediatrics, 36, 117-130. https://doi.org/10.3109/01942638.2015.1040574

Gándara-Gafo, B., Santos-del Riego, S., \& Muñiz, J. (2016). Adaptación cultural, validación y valores de referencia del Adolescent/Adult Sensory Profile en España [Cultural adaptation, validation, and reference values for the Adolescent/Adult Sensory Profile in Spain] (Unpublished doctoral dissertation). Universidade da Coruña, A Coruña, Spain.

Hambleton, R. K., \& Patsula, L. (1998). Adapting tests for use in multiple languages and cultures. Social Indicators Research, 45, 153-171.

International Test Commission. (2005). ITC guidelines for translating and adapting tests. Retrieved from https://www.intestcom.org/files/guideline_test_adaptation.pdf

Kinnealey, M., \& Oliver, B. (2002). Adult Sensory Questionnaire. Unpublished instrument. 
Neuman, A., Greenberg, D. F., Labovitz, D. R., \& Suzuki, L. A. (2004). Cross-cultural adaptation of the Sensory Profile: Establishing linguistic equivalency of the Hebrew version. Occupational Therapy International, 11, 112-130. https://doi.org/10.1002/oti.201

Ramada-Rodilla, J. M., Serra-Pujadas, C., \& Delclós-Clanchet, G. L. (2013). Adaptación cultural y validación de cuestionarios de salud: Revisión y recomendaciones metodológicas [Cultural adaptation and validation of health questionnaires: Review and methodological recommendations]. Salud Pública de México, 55, 57-66. https://doi.org/10.1590/S0036-36342013000100009

Román-Oyola, R., \& Reynolds, S. E. (2010). Validating the response process of the Spanish version of the Short Sensory Profile: A pilot study using cognitive interviews. Journal of Occupational Therapy, Schools, and Early Intervention, 3, 197-206. https://doi.org/10.1080/19411243.2010.515189

Suarez-Balcazar, Y., Rodawoski, J., Balcazar, F., Taylor-Ritzler, T., Portillo, N., Barwacz, D., \& Willis, C. (2009). Perceived levels of cultural competence among occupational therapists. American Journal of Occupational Therapy, 63, 498-505. https://doi.org/10.5014/ajot.63.4.498

Google Scholar

Weinreich, U. (1979). Languages in contact: Findings and problems. New York: Linguistic Circle of New York. https://doi.org/10.1515/9783110802177

Willis, G. B. (2005). Cognitive interviewing: A tool for improving questionnaire design. Thousand Oaks, CA: Sage. 